\title{
Quantitative Phase Imaging of Thin Film Surface
}

\author{
E. TIRYAKI ${ }^{a}$, Ö. KOCAHAN ${ }^{b, *}$ AND S. ÖZDER ${ }^{c}$ \\ ${ }^{a}$ Department of Physics, School of Graduate Studies, \\ Canakkale Onsekiz Mart University, Canakkale, Turkey \\ ${ }^{b}$ Department of Physics, Faculty of Arts and Sciences, \\ Tekirdag Namik Kemal University, Tekirdag, Turkey \\ ${ }^{c}$ Department of Physics, Faculty of Arts and Sciences, \\ Canakkale Onsekiz Mart University, Canakkale, Turkey
}

Received: 03.05.2021 \& Accepted: 05.10.2021

Doi: $10.12693 /$ APhysPolA.140.281

*e-mail: okocahan@nku.edu.tr

In this study, the white light diffraction phase microscopy and the generalized Morse wavelet are proposed to achieve practical and precise measurement of a thin film surface. The white light diffraction phase microscopy provides low speckle noise and single-shot measurement, and thus it has been used to produce an image with interference fringes from the surface of a thin film. Relying on produced interferogram, quantitative phase information of the thin film surface has been obtained using the continuous wavelet transform. In the calculation of the quantitative phase, in many studies the continuous wavelet transform method with different wavelets is preferred. The Morlet wavelet is a commonly used one with a fixed resolution. An alternative approach is proposed using the generalized Morse wavelet capable of controlling the resolution. It has an additional advantage of varying the two parameters, thus improving the sensitivity of phase calculation. Results of the generalized Morse wavelet were compared with the Morlet and Paul wavelets which also have one varying parameter. For the determination of the thin film surface profile, besides the white light diffraction phase microscopy, surfaces have been investigated by a Dektak stylus profilometer and a scanning electron microscope. In this way, it was possible to observe the difference between the most commonly used methods with regard to the imaging of thin film surfaces. The application of the white light diffraction phase microscopy with the generalized Morse wavelet was compared with the common microscopy techniques for studying thin film surfaces, and experimental results were discussed at the end of the study.

topics: thin film surface, diffraction phase microscopy, generalized Morse wavelet, fringe analysis

\section{Introduction}

The detailed surface roughness information of an optical thin film is crucial for the production of optical devices. This characteristic information has an important role to optimize the optical coating systems. There are various methods to measure a surface profile $[1,2]$, such as the scanning electron microscopy (SEM) [3, 4], the atomic force microscopy (AFM) [5,6], the classical stylus profilometry technique $[7,8]$, and the interferometric method [9]. Stylus profilometry is widely used for the surface roughness, but it can cause surface damage. On the other hand, AFM and SEM are quite expensive instruments. The thin film surface analysis by these tools is extremely complex and it consumes too much time.

Non-contact techniques for rapidly and accurately mapping surfaces on a micro- or nanoscale are one of the most remarkable fields in optoelectronics industry [10]. Interferometry is one of the useful tools which provide information on the surface morphology with high accuracy and in a non-destructive way [11]. Interferometric microscopy is based on the interference of the sample beam and an off-axis reference beam to form a fringe [12-14]. Many interferometric techniques have been used to study the dynamics and morphology of different samples [10, 15]. In all of these applications, it is necessary to investigate the sensitivity limits and their relationship to the setup [16].

The measurement accuracy in optical interferometry is essential. Several methods for the determination of a 3D surface profile from fringe patterns were proposed [17-19]. In this study, the white light diffraction phase microscopy (WDPM) is used to obtain interference fringes for the surface of the thin film. This method provides a low speckle noise and a single shot measurement with white light [20,21]. The image of the thin film surface with the fringe pattern is recorded and the phase values are calculated from this image by using the continuous wavelet transform (CWT). 
The choice of the analyzing wavelet for the CWT method is important to achieve more precise results [22]. The commonly used Morlet wavelet meets the condition of admissibility with a fixed spatial frequency selected as 5 or 6 . Therefore, it has a maximum fixed resolution and a minimum uncertainty [18]. When the resolution is not compatible with the repetition frequency of the analyzed signal, the Morlet wavelet cannot produce successful results. On the other hand, the Paul wavelet has the ability to control the resolution of the analyzing wavelet by one variable parameter. Because of this degree of freedom, the Paul wavelet can better detect the local characteristics of the fringe pattern, which results in a precise measurement [23]. The generalized Morse wavelet (GMW) is preferred in this work as the analyzing wavelet which has two degrees of freedom with two variable parameters [24]. These parameters allow to control the resolution so that better results can be obtained.

The aim of this study is to determine with high accuracy the quantitative surface roughness information of a CdS film. The WDPM setup was used for imaging the surface quantitatively. Also, zero order GMW was used as a mother wavelet in the CWT technique to retrieve the phase with high precision. Moreover, SEM images and Dektak stylus profilometer measurements were used to determine the thickness of the thin film and for the visualization of the surface. In this way, it was possible to observe the differences in terms of precision and implementation between the most commonly used methods with regard to the imaging of thin film surfaces.

\section{GMW method}

The mathematical expression of a signal corresponding to any $y$-pixel (row) of an interferogram is

$$
h(x)=I_{0}\left[1+V(x) \cos \left(2 \pi f_{0}+\varphi(x)\right)\right],
$$

where $V(x)$ is the visibility of the fringe, $I_{0}(x)$ is the background intensity, $f_{0}$ is the spatial carrier frequency in the $x$ direction, and $\varphi(x)$ is the heightmodulated phase of the fringe. To recover the phase, $f_{0}$ has to meet the following condition [25]

$$
2 \pi f_{0}>\left|\frac{\mathrm{d} \varphi}{\mathrm{d} x}\right|_{\max } .
$$

The one-dimensional CWT is defined as [26]

$$
\operatorname{CWT}(a, b)=\int_{-\infty}^{\infty} \mathrm{d} x h(x) \Psi_{a, b}^{*}(x),
$$

where $\Psi_{a, b}^{*}(x)$ is the complex conjugate of the analyzing wavelet, i.e.,

$$
\Psi_{a, b}^{*}(x)=\frac{\Psi\left(x-\frac{b}{a}\right)}{\sqrt{a}},
$$

where $a(a>0)$ is the scale parameter and $b$ is the translation parameter. By using Parseval's identity, the CWT formula given in (3) can be rewritten as

$$
\operatorname{CWT}(a, b)=\sqrt{a} \int_{-\infty}^{\infty} \mathrm{d} \alpha \hat{\Psi}^{*}(a \alpha) \hat{H}(\alpha) \mathrm{e}^{\mathrm{i} b \alpha},
$$

where $\hat{\Psi}(a \alpha)$ and $\hat{H}(\alpha)$ are the Fourier transforms of $(1 / \sqrt{a}) \Psi(x-b / a)$ and $h(x)$, respectively. The new form of CWT enables us to use the fast Fourier transform (FFT) algorithm for faster processing.

In this work, zero order GMW is selected as the mother wavelet. The description of its analyzing wavelet form in the Fourier domain [27, 28] is given as

$$
\hat{\Psi}_{\beta, \gamma}(a \alpha)=U(\alpha) N_{\beta, \gamma}(a \alpha)^{\beta} \exp \left(-(a \alpha)^{\gamma}\right) .
$$

Here, $U(\alpha)$ is the Heaviside step function, $\beta$ and $\gamma$ are two varying parameters and $N_{\beta, \gamma}=$ $2[\exp (1) \gamma / \beta]^{\beta / \gamma}$ is the normalization constant. We can now substitute (6) and the Fourier transform of (1) into the new form of the CWT equation, additionally noting that $\hat{\Psi}_{\beta, \gamma}(a \alpha)=0$ for $(a \leq 0)$. As a result, CWT with GWM is acquired as

$$
\begin{aligned}
& \operatorname{CWT}(a, b)=2 \pi I_{0}(b) V(b) a^{\beta+\frac{1}{2}}\left(\frac{\gamma}{\beta}\right)^{\frac{\beta}{\gamma}}\left(f_{0}+\frac{\varphi^{\prime}}{2 \pi}\right)^{\beta} \exp \left(\frac{\beta}{\gamma}-a\left(f_{0}+\frac{\varphi^{\prime}}{2 \pi}\right)^{\gamma}\right) \\
& \quad \times \exp \left(\mathrm{i}\left(\varphi(b)-b \varphi^{\prime}(b)+b f_{0}+b \frac{\varphi^{\prime}}{2 \pi}\right)\right)
\end{aligned}
$$

The wrapped phase distribution can be extracted from CWT by using the following equation

$$
\varphi(b)=\tan ^{-1}\left(\frac{\operatorname{Im}(\mathrm{CWT})}{\operatorname{Re}(\mathrm{CWT})}\right) .
$$

To retrieve the real phase, MATLAB unwrapping code was chosen in this study [29]. By applying the unwrapping procedure, correct phase information is found for each row.

\section{Simulation and experimental work}

For such applications, an important step is to choose the mother wavelet. In this respect, the localization properties of wavelets can be evaluated. The uncertainty values of the wavelets were compared in Table I [24]. The results in Table I indicate that the phase calculation sensitivity varies with an extra degree of freedom. 


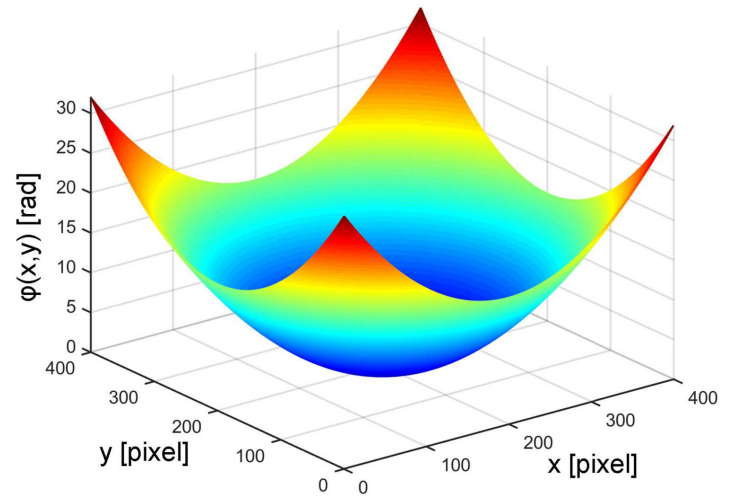

Fig. 1. The simulated phase function given by (9).

TABLE I

Uncertainties for given $\beta$ and $\gamma$ values of GMW as well as uncertainty of the Morlet and $m$-th Paul wavelets.

\begin{tabular}{|c|c|c|c|c|c|}
\hline \multicolumn{6}{|c|}{ GMW } \\
\hline \multirow{2}{*}{$\gamma$} & \multicolumn{5}{|c|}{$\beta$} \\
\hline & 1 & 3 & 5 & 7 & 10 \\
\hline 1 & 0.86 & 0.59 & 0.55 & 0.54 & 0.53 \\
\hline 3 & 0.53 & 0.50 & 0.50 & 0.50 & 0.50 \\
\hline 5 & 0.53 & 0.51 & 0.51 & 0.51 & 0.50 \\
\hline 7 & 0.56 & 0.53 & 0.52 & 0.52 & 0.51 \\
\hline 10 & 0.61 & 0.57 & 0.55 & 0.54 & 0.53 \\
\hline \multicolumn{6}{|c|}{ Paul } \\
\hline \multicolumn{6}{|l|}{$m$} \\
\hline 1 & \multicolumn{5}{|c|}{0.86} \\
\hline 20 & \multicolumn{5}{|c|}{0.51} \\
\hline 40 & \multicolumn{5}{|c|}{0.50} \\
\hline 60 & \multicolumn{5}{|c|}{0.50} \\
\hline 80 & \multicolumn{5}{|c|}{0.50} \\
\hline \multicolumn{6}{|c|}{ Morlet } \\
\hline & & & 0.50 & & \\
\hline
\end{tabular}

We tested the method described in Sect. 2 and used the following phase expression

$$
\varphi(x, y)=0.0004\left[(x-200)^{2}+(y-200)^{2}\right] .
$$

The fringe pattern (1) and the phase given in (9) were calculated for given values of $I_{0}(x)=1.0$, $V(x)=1.0$ and $f_{0}=0.2$ per pixel. The results are shown in Fig. 1.

The height modulated fringe pattern shown in Fig. 2 was analyzed with the newly proposed CWT method and the correct phases were calculated by unwrapping the wrapped phases. As seen in Table I, GMW satisfies the minimum uncertainty at $\gamma=3$ and 10 when $\beta=3,5,7,10$, so the analysis process is repeated for each of these values. The absolute phase error was determined and demonstrated in Fig. 3, and it is equal to the difference between the test and the calculated phases.
The two variable parameters of GMW were selected as $(\gamma, \beta)=(3,10)$ in accordance with this error analysis. Simulations were achieved also with the Morlet wavelet and the Paul wavelet for comparison and the absolute phase error was shown in Fig. 4.

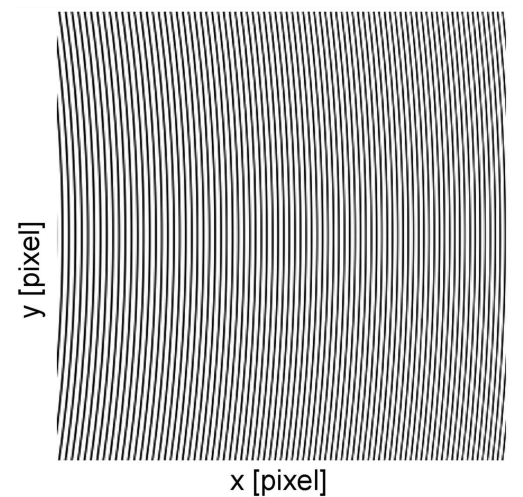

Fig. 2. The height-modulated fringe pattern.

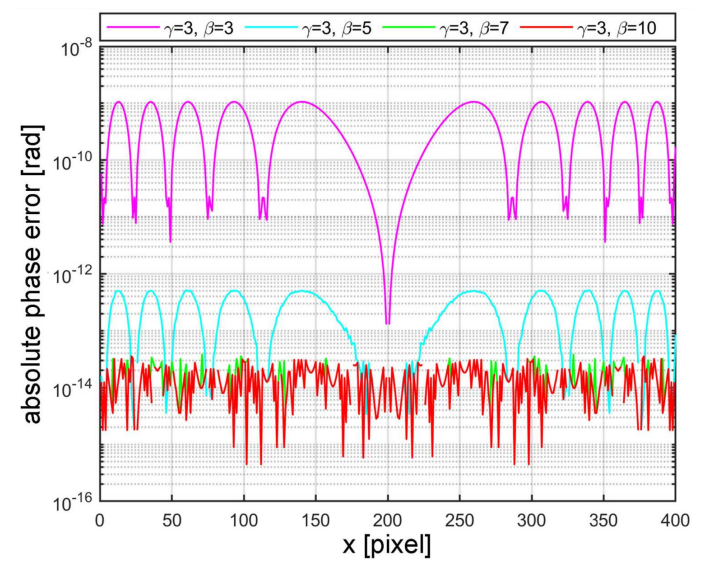

Fig. 3. The absolute phase error in logarithmic scale, for the line $y=200$ by taking $\gamma=3$ when $\beta=3$ (magenta), $\beta=5$ (cyan), $\beta=7$ (green), $\beta=10$ (red) by GMW.

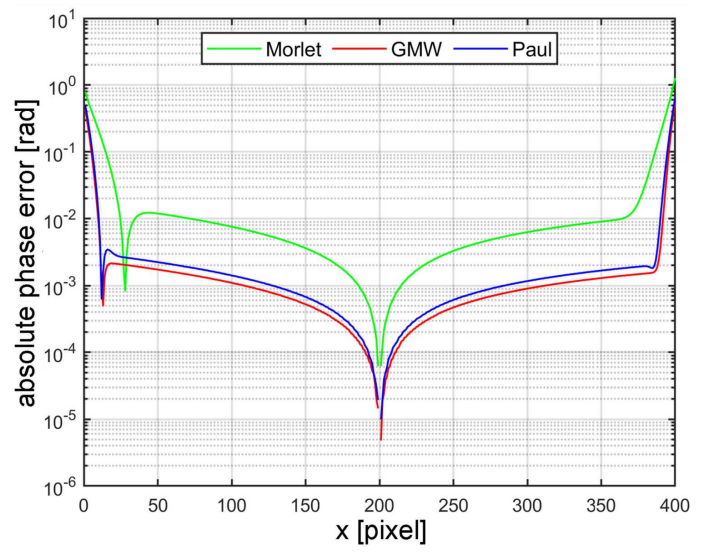

Fig. 4. The absolute phase error: $\operatorname{GMW}(\gamma, \beta)$ $=(3,10)($ red $)$, the Morlet wavelet (green) and the Paul wavelet $(m=40)$ (blue). 


\subsection{WDPM method}

An experimental study was conducted to verify the validity of the method described above. The experimental setup and schematic diagram of WDPM are shown in Fig. 5. The WDPM has a huge advantage that it is a non-contact surface measurement technique and a single-shot measurement is possible.

The system configuration consists of a combination of a Mach-Zehnder interferometer and an inverted microscope with a halogen lamp. At first, an Axio Observer A1 Zeiss Inverted microscope with $20 \times$ and $40 \times$ objectives is set for a clear image of the sample. Amplitude grating (110 groves per $\mathrm{mm}$ ) is located in front of the microscope to generate diffraction orders. These diffraction order beams carry a highly sensitive phase information of the specimen. With a $60 \mathrm{~mm}$ focal length lens, zero and first order rays are passed through the pinhole. This pinhole consists of two apertures with the diameters of $0.2 \mathrm{~mm}$ and $5 \mathrm{~mm}$. The zero and first order components play the role as reference and specimen images. With the second lens (150 mm focal length), the interferogram is formed by the interference of these two beams on the sCMOS camera plane [24]. Thus, the images with interference fringes of the specimen and reference are recorded for the analysis.

First of all, a reference image with the camera in the resulting image plane is recorded from an uncoated part of the sample thin film. Then, an interferometric image is created and recorded for a coated part of the thin film placed in the microscope. The interferometer enlarges the sample and reference image by $f_{2} / f_{1}=2.5$ times. Considering
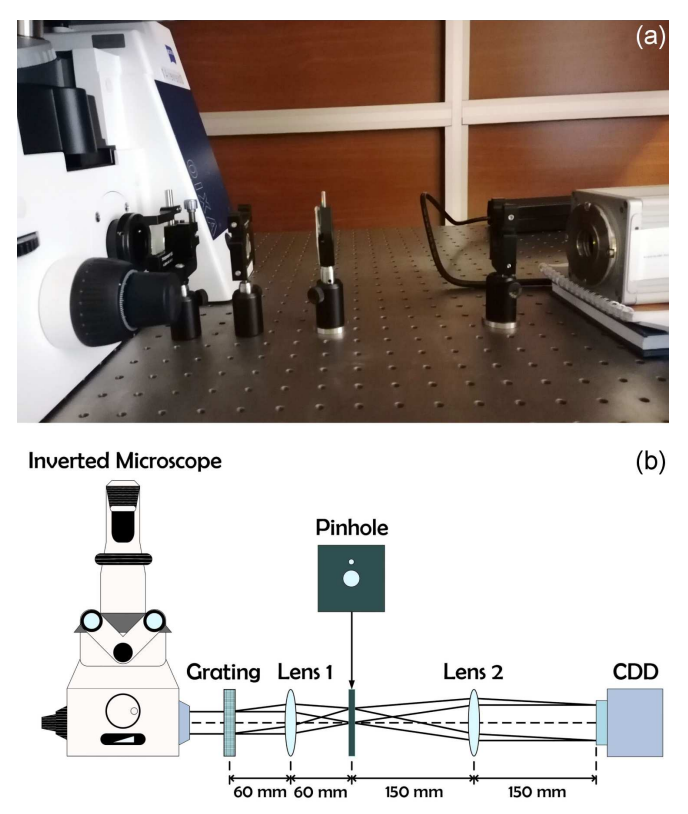

Fig. 5. The experimental setup (a) and schematic diagram of WDPM (b).
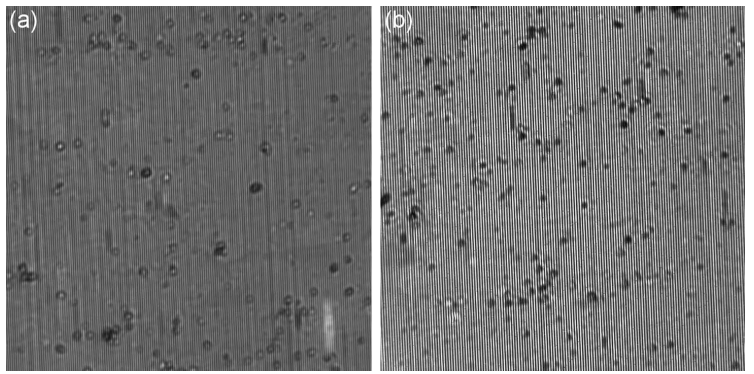

Fig. 6. Images with interference fringes of the CdS thin film surface with (a) $20 \times$ objective, (b) $40 \times$ objective.
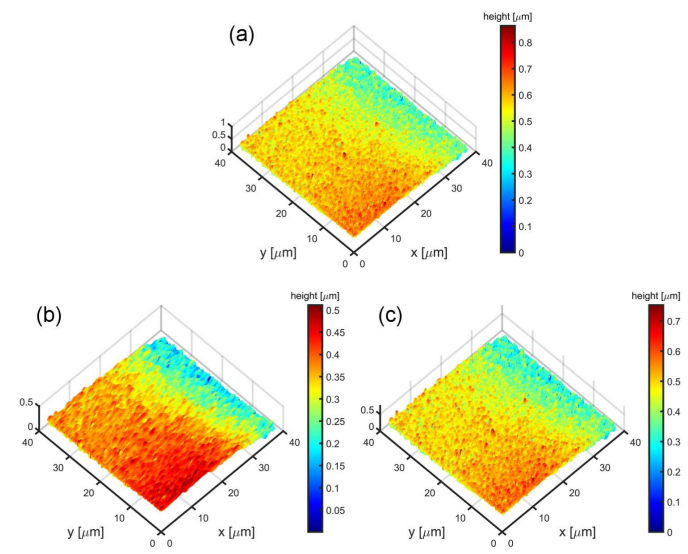

Fig. 7. The surface profile of the CdS thin film with $20 \times$ objective by using (a) GMW $((\gamma, \beta)=(3,10)),(\mathrm{b})$ the Morlet wavelet, (c) the Paul wavelet $(m=40)$.

the magnification of the objectives, one can obtain a 100 times magnification with the $40 \times$ lens and a 50 times magnification with the $20 \times$ lens.

The plane where the interference pattern occurs is the camera plane which is the last element of the WDPM setup. A Hamamatsu ORCA Flash 4.0 CMOS camera was used in the experimental setup. The sCMOS image sensor in this camera performs both low noise (1.0 electron (median) and 1.6 electron (rms)) and high-speed reading $\left(2048 \times 2048\right.$ pixels $^{2}$ and 100 frames/s $)$ simultaneously, and the exposure time is $1 \mathrm{~ms}$. The number of pixels of the camera used is $2048 \times 2048$, its effective area is $13.312 \mathrm{~mm}^{2}$ and the pixel size is $6.5 \mu \mathrm{m}$. Considering the 100 times magnification value obtained with the interferometer, for the $40 \times$ lens, the size of the pixel in the resulting image was $0.065 \mu \mathrm{m}$. The observed area was $0.085 \mathrm{~mm}^{2}$ in the image for this objective. When using the $20 \times$ objective, this area becomes $0.27 \mathrm{~mm}^{2}$.

For this study, the CdS thin film surface and reference images have been taken by WDPM, in the middle of the sample, as shown in Fig. 6. At first, reference and specimen images were analyzed separately to find the wrapped phase distributions. Then, they were unwrapped and subtracted from 

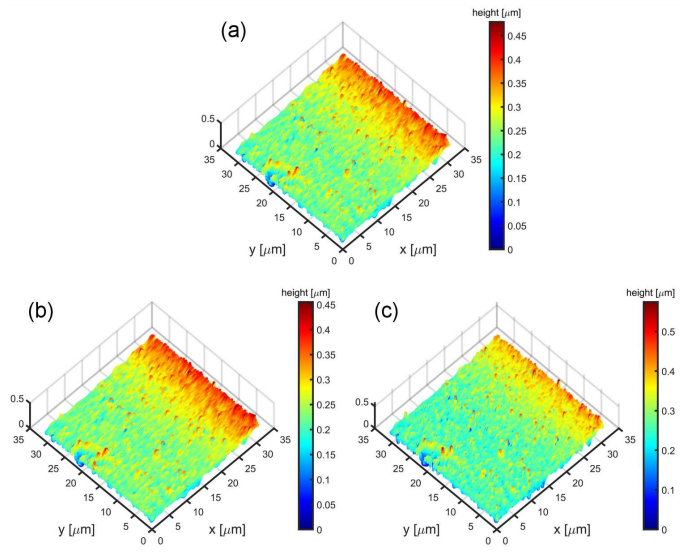

Fig. 8. The surface profile of the CdS thin film with $40 \times$ objective by using (a) GMW $((\gamma, \beta)=$ $(3,10))$, (b) the Morlet wavelet, (c) the Paul wavelet $(m=40)$.

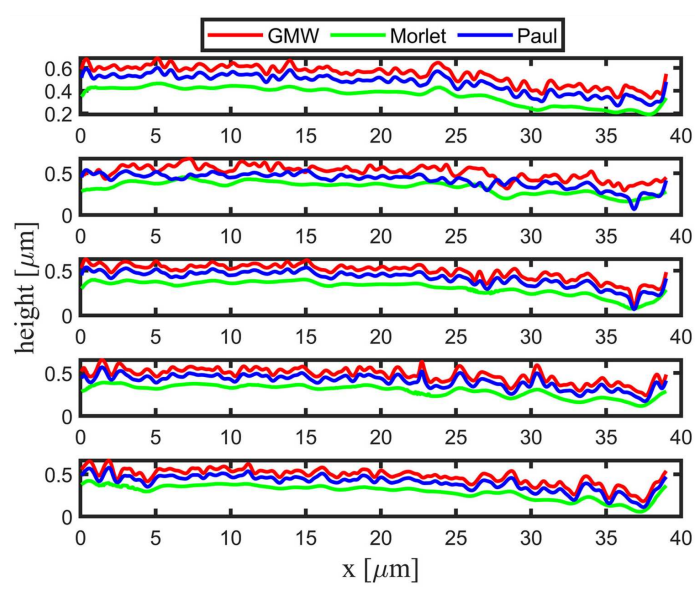

Fig. 9. The height values of the thin film surface (20× objective) for the lines $y=6.5,13,19.5,26$, and $29.25 \mu \mathrm{m}$ (from top to bottom) respectively, by the CWT method with zero order $\operatorname{GMW}((\gamma, \beta)=$ $(3,10))$ (red), the Morlet (green), the Paul wavelet $(m=40)$ (blue).

each other to retrieve the correct phase information of the thin film surface. The height values were determined from these phases [30] as $h(x, y)=$ $\lambda \varphi(x, y) /\left[2 \pi\left(n_{2}-n_{1}\right)\right]$. Here, $\lambda=550 \mathrm{~nm}$ is the center wavelength of the halogen lamp used in the microscope, whereas $n_{2}=2.6$ and $n_{1}=1$ are the refractive index of the $\mathrm{CdS}$ thin film at that wavelength and the surrounding medium, respectively. The retrieved height values of the $\mathrm{CdS}$ thin film surface, calculated from the images in Fig. 6 by using the CWT phase method with zero order GMW $((\gamma, \beta)=(3,10))$, were indicated in Figs. 7 and 8 . Also, by using the Morlet and Paul wavelets, surface profiles were calculated and given in these figures. The comparison between the height values for the lines $y=6.5,13,19.5,26$ and $29.25 \mu \mathrm{m}$ are demonstrated in Figs. 9 and 10 for the $20 \times$ and $40 \times$ objectives, respectively.

\subsection{SEM imaging}

Structural, morphological and topographic properties, elemental analysis and mapping of the sample surfaces can be examined by SEM. It is possible to display them at different magnifications. However, as a result of surface imaging, SEM gives a two-dimensional image with scale information. In this respect, SEM has a disadvantage as compared to the surface profilometers. The CdS thin film surface and thickness, examined in a Quanta FEG SEM in NABILTEM (Central Research

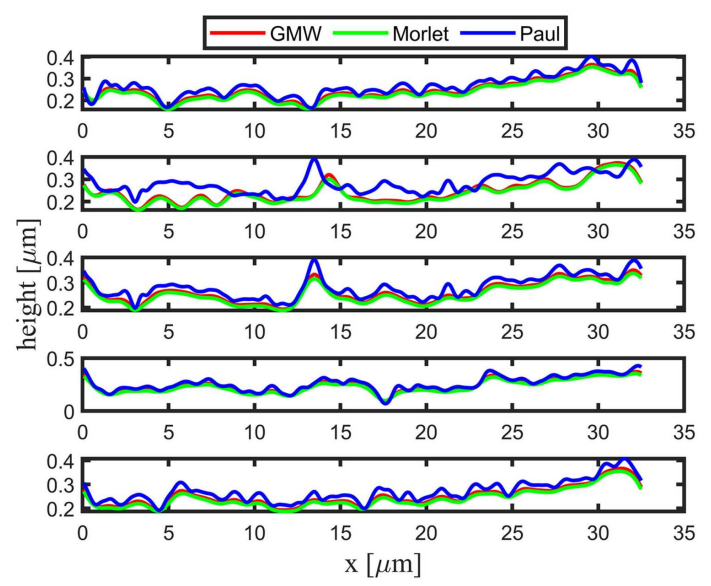

Fig. 10. The height values of the thin film surface $(40 \times$ objective) for the lines $y=29.25,26,19.5$, 13 , and 6.5 (from top to bottom) respectively, by the CWT method with zero order GMW $((\gamma, \beta)=$ $(3,10))$ (red), the Morlet (green), the Paul wavelet $(m=40)$ (blue).
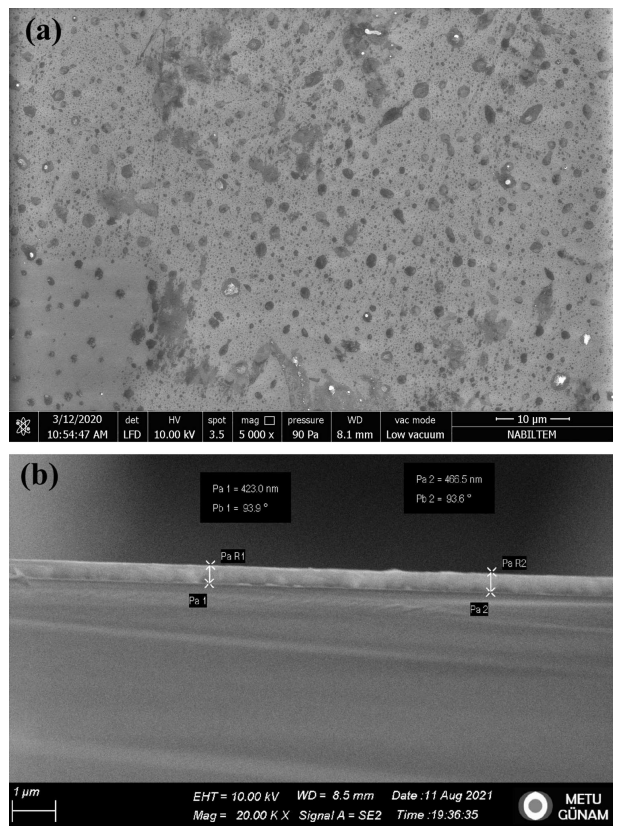

Fig. 11. SEM images taken in the middle part of the CdS thin film: (a) surface, (b) cross-sectional field. 

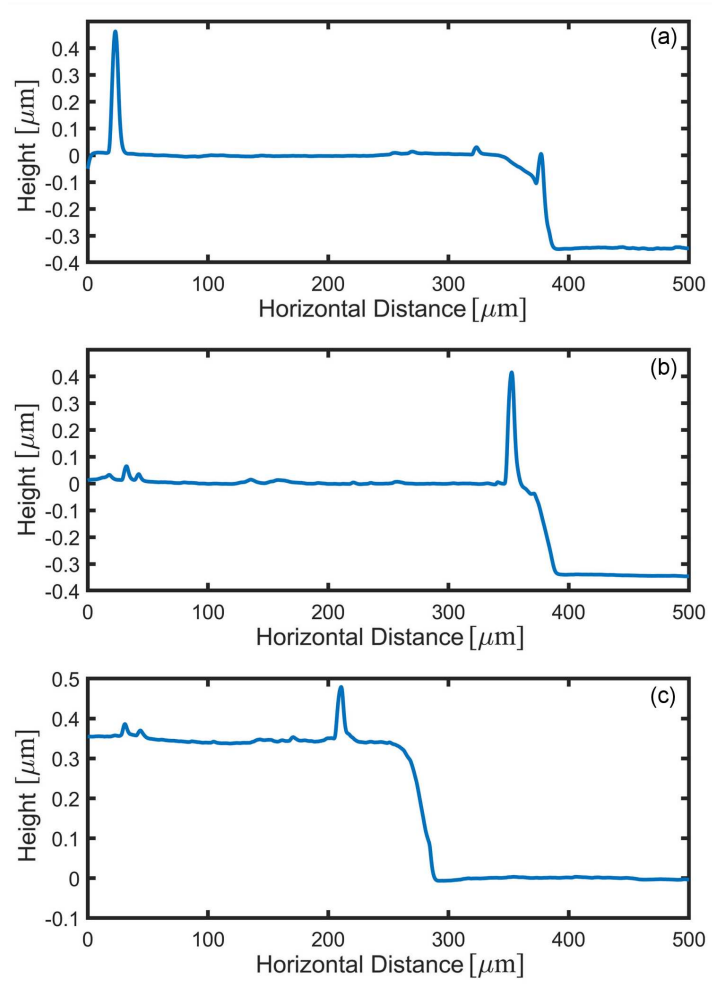

Fig. 12. The surface roughness obtained by the Dektak surface profilometer for three different regions of the thin film surface.

Laboratory) at Tekirdă̆ Namı Kemal University and GUNAM (Center for Solar Energy Research and Applications) at Middle East Technical University, are shown in Fig. 11.

\subsection{Dektak measurement}

A Dektak surface profilometer, a surface contact measurement technique, is an instrument to measure the vertical profile of samples, thin film thickness, and other topographical features [31, 32]. However, this measurement can cause surface damage because of the contact with the surface. The thin film thickness of CdS was measured around $400 \mathrm{~nm}$ in the middle and $350 \mathrm{~nm}$ at the side of the film by the Veeco Dektak $6 \mathrm{~m}$ stylus profilometer at GUNAM, Middle East Technical University. The obtained profiles, which show the surface roughness, are given in Fig. 12.

\section{Conclusions}

To determine the phase distribution of the CdS thin film surface, the CWT method with zero order GMW was used. According to the simulations and Fig. 3, the GMW method for $(\gamma, \beta)=(3,10)$ provides the minimum absolute phase error. As can be seen from Table I, it has such an advantage that GMW controls the resolution with the extra degree of freedom. Thanks to this feature, better localization is possible.
For comparison of phase calculation methods, the Morlet and Paul wavelets were used to calculate the phase distribution. GMW is analytical, so it consequently performs better in comparison to Morlet. Two degrees of freedom also help in comparison to the Paul wavelet. Simulation results shown in Fig. 4 indicate that the GMW method is more accurate for the phase calculation according to the Morlet wavelet, however, the absolute phase error for the Paul wavelet and GMW are close to each other. Therefore, it is possible to say that controlling the resolution brings accurate phase calculation.

Mapping surfaces on microscale quantitatively have been achieved by the WDPM setup in a nondestructive way (Figs. 6-8). In Fig. 6, fringe visibility seems better for the $40 \times$ objective lens in comparison with $20 \times$ because of the magnifications. The magnification of 100 times was obtained with the $40 \times$ lens and 50 times magnification — with the $20 \times$ lens. Besides, the thin film surface has been imaged by Dektak and SEM. In this way, surface profiles could be determined by different microscopy techniques. It is possible to observe different scales with SEM. However, Figs. 7 and 8 give more information about the surface roughness of the film according to the SEM image (Fig. 11a). Unlike Fig. 11, Figs. 7 and 8 are in a three-dimensional and dynamic form. Moreover, for the SEM image, the measurement of the size and roughness of the surface is not quantitative. Dektak measurements have given the film thickness as $400 \mathrm{~nm}$ in the middle and $350 \mathrm{~nm}$ at the side of the sample, but this measurement has caused damage at the surface of the thin film. Surface roughness, observed with the Dektak surface profilometer for three lines (Fig. 12), is compatible with Figs. 9 and 10. The height values obtained by all methods are in harmony.

From WDPM images, the calculated surface profiles of the thin film compared in Figs. 9 and 10 demonstrate that GMW has a high precision since it has two degrees of freedom. The obtained results are consistent with the simulation study.

The 3D surface profile of the thin film was determined by zero order GMW which was improved as a tool to be able to determine the surface roughness of the thin film. Results are consistent with the Dektak measurements and SEM images. Experimental work shows that the WDPM setup is very convenient for this kind of measurements. The stable common path interferometer provides robustness to outside vibrations; hence nanoscale measurements are possible. The WDPM measurement and GMW phase calculation techniques have advantages in terms of non-contact, quantitative, and 3D precise measurement. Spatial and frequency swapping feature allows more accurate measurements and calculations with the GMW method. This reveals how appropriate it is to choose GMW as the analyzing wavelet for this kind of works. 


\section{Acknowledgments}

This work was supported by the Tekirda $\breve{g}$ Namık Kemal University Scientific Research Project Commission (NKUBAP.01.GA.18.148) and the Turkish Scientific and Technical Research Council (TUBITAK-MFAG No 120F325).

\section{References}

[1] D.J. Whitehouse, Meas. Sci. Technol. 8, 955 (1997).

[2] M.R. Nenkov, T.G. Pencheva, Cent. Eur. J. Phys. 6, 332 (2008).

[3] T. Mathia, H. Zahouani, J. Rousseau, J.C. Le Bosse, Int. J. Mach. Tools Manufact. 35, 195 (1995).

[4] Z.W. Kowalski, Acta Phys. Pol. A 120, 70 (2011).

[5] M. Zhong, F. Chen, C. Xiao, Y. Wei, Opt. Lasers Eng. 88, 243 (2017).

[6] S. Çörekçí, K. Kızılkaya, T. Asar, M.K. Öztürk, M. Çakmak, S. Özçelík, Acta Phys. Pol. A 121, 247 (2012).

[7] R. Kulkarni, P. Rastogi, Opt. Lasers Eng. 87, 1 (2016).

[8] A. Gunay, B. Sagbas, M.N. Durakbasa, Acta Phys. Pol. A 125, 484 (2014).

[9] C.Y. Poon, B. Bhushan, Wear 190, 76 (1995).

[10] S.K. Debnath, M.P. Kothiyal, J. Schmit, P. Hariharan, Opt. Express 14, 4662 (2006).

[11] W.B. Ribbens, Appl. Opt. 8, 2173 (1969).

[12] D.J. Lee, A.M. Weiner, Opt. Express 22, 266 (2014).

[13] Y. Hwang, S. Yoon, J.-H. Kim, S. Kim, H.-J. Pahk, Opt. Lasers Eng. 46, 179 (2008).

[14] Y.-S. Ghim, S.-W. Kim, Opt. Express 14, 11885 (2006).

[15] G. Popescu, Quantitative Phase Imaging of Cells and Tissues, McGraw-Hill, 2011.
[16] P. Hosseini, R.-J. Zhou, Y.-H. Kim, Ch. Peres, A. Diaspro, C.F. Kuang, Z. Yaqoob, P.T.C. So, Opt. Lett. 41, 1656 (2016).

[17] M. Takeda, K. Mutoh, Appl. Opt. 22, 3977 (1983).

[18] A. Dursun, S. Özder, F.N. Ecevit, Meas. Sci. Technol. 15, 1768 (2004).

[19] S. Özder, Ö. Kocahan, E. Coşkun, H. Göktaş, Opt. Lett. 32, 591 (2007).

[20] B. Bhaduri, H. Pham, M. Mir, G. Popescu, Opt. Lett. 37, 1094 (2012).

[21] H.V. Pham, C. Edwards, L.L. Goddard, G. Popescu, Appl. Opt. 52, A97 (2013).

[22] A. Drzewiecki, P.B. Sczaniecki, Acta Phys. Pol. A 108, 73 (2005).

[23] E. Coşkun, S. Özder, J. Opt. Soc. Am. B 28, 2974 (2011).

[24] O. Kocahan, E. Tiryaki, E. Coskun, S. Ozder, Meas. Sci. Technol. 29, 035203 (2018).

[25] M. Afifi, A. Fassi-Fihri, M. Marjane, K. Nassim, M. Sidki, S. Rachafi, Opt. Commun. 211, 47 (2002).

[26] C. Torrence, G.P. Compo, Bull. Am. Meteorol. Soc. 79, 61 (1998).

[27] J.M. Lilly, S.C. Olhede, IEEE Trans. Inf. Theory 56, 4135 (2010).

[28] J.M. Lilly, S.C. Olhede, IEEE Trans. Signal Process. 60, 6036 (2012).

[29] D.C. Ghiglia, D. Mark, Two-Dimensional Phase Unwrapping: Theory, Algorithms, and Software, Vol. 2020, Wiley, 2020.

[30] C. Edwards, R.J. Zhou, S.-W. Hwang et al., Appl. Opt. 53, G33 (2014).

[31] Integrated Nanosystems Research Facility (INRF), "Dektak 3 Profilometer", 2020.

[32] G.Q. Zhou, H. Ding, L. Zhu et al., J. En. Chem. 47, 180 (2020). 